\title{
Economic evaluation of first-line and maintenance treatments for advanced non-small cell lung cancer: a systematic review
}

This article was published in the following Dove Press journal:

ClinicoEconomics and Outcomes Research

15 December 2014

Number of times this article has been viewed

\section{Christos Chouaïd' \\ Perinne Crequit ${ }^{2}$ \\ Isabelle Borget ${ }^{3}$ \\ Alain Vergnenegre ${ }^{4}$}

'Service de Pneumologie et de Pathologie Professionnelle, Centre Hospitalier Intercommunal Créteil et Université de Paris Est Créteil, Paris, France; ${ }^{2}$ Service de Pneumologie, Hôpital Tenon, Assistance PubliqueHôpitaux de Paris, Paris, France; ${ }^{3}$ Service de Biostatistique et d'Epidémiologie, Institut Gustave Roussy, Villejuif, France; ${ }^{4}$ Unité d'Oncologie Thoracique et Cutanée, Centre Hospitalier Universitaire Limoges, Limoges, France
Correspondence: Christos Chouaïd

Service de pneumologie,

$\mathrm{CHI}$ Créteil, 40 avenue de verdun,

94010 Créteil, France

Tel +33 I 57022070

Fax +33 I 57022059

Email christos.chouaid@chicreteil.fr
Abstract: During these last years, there have been an increased number of new drugs for non-small cell lung cancer (NSCLC), with a growing financial effect on patients and society. The purpose of this article was to review the economics of first-line and maintenance NSCLC treatments. We reviewed economic analyses of NSCLC therapies published between 2004 and 2014. In first-line settings, in unselected patients with advanced NSCLC, the cisplatin gemcitabine doublet appears to be cost-saving compared with other platinum doublets. In patients with nonsquamous NSCLC, the incremental cost-effectiveness ratios (ICERs) per life-year gained (LYG) were $\$ 83,537, \$ 178,613$, and more than $\$ 300,000$ for cisplatin-pemetrexed compared with, respectively, cisplatin-gemcitabine, cisplatin-carboplatin-paclitaxel, and carboplatinpaclitaxel-bevacizumab. For all primary chemotherapy agents, use of carboplatin is associated with slightly higher costs than cisplatin. In all the analysis, bevacizumab had an ICER greater than $\$ 150,000$ per quality-adjusted life-year (QALY). In epidermal growth factor receptor mutated advanced NSCLC, compared with carboplatin-paclitaxel doublet, targeted therapy based on testing available tissue yielded an ICER of \$110,644 per QALY, and the rebiopsy strategy yielded an ICER of $\$ 122,219$ per QALY. Compared with the triplet carboplatin-paclitaxelbevacizumab, testing and rebiopsy strategies had ICERs of \$25,547 and \$44,036 per QALY, respectively. In an indirect comparison, ICERs per LYG and QALY of erlotinib versus gefitinib were $\$ 39,431$ and $\$ 62,419$, respectively. In anaplastic lymphoma kinase-positive nonsquamous advanced NSCLC, the ICER of first-line crizotinib compared with that of chemotherapy was $\$ 255,970$ per QALY. For maintenance therapy, gefitinib had an ICER of \$19,214 per QALY, erlotinib had an ICER of \$127,343 per LYG, and pemetrexed had an ICER varying between $\$ 183,589$ and $\$ 205,597$ per LYG. Most recent NSCLC strategies are based on apparently no cost-effective strategies if we consider an ICER below \$50,000 per QALY an acceptable threshold. We need, probably on a countrywide level, to have a debate involving public health organizations and pharmaceutical companies, as well as clinicians and patients, to challenge the rising costs of managing lung cancer.

Keywords: lung cancer, costs, economics, cost-effectiveness, evaluation

\section{Introduction}

During these last years, the number of oncology-related investigational new drug applications has increased, with a growing financial effect on patients and society. Cancer care costs are escalating at a rate of $15 \%$ per year, which is nearly three times the increase in overall health care spending. ${ }^{1}$ Lung cancer is a leading cause of cancerrelated deaths, with an estimated 219,440 new cases and 159,390 deaths in 2009 in the United States. The economic cost of lung cancer is high, estimated at $\$ 9$ billion per year. Non-small cell lung cancer (NSCLC) makes up approximately $85 \%$ of lung cancer cases, 
and around $65 \%$ of patients are diagnosed with advanced stages, with estimated 2 and 5 year survival rates of $15 \%$ and $2 \%$, respectively. The main subtypes of NSCLC are squamous cell carcinoma (33\%), adenocarcinoma (61\%), and large cell carcinoma (6\%). Because of the incidence, severity, and rising costs of this illness, it is becoming increasingly important to deliver consistent, high-quality, cost-effective care for NSCLC. ${ }^{2-5}$ The purpose of this article is to review the economics of first-line and maintenance treatments for NSCLC. After recalling the current management of NSCLC, we review the cost-effectiveness of advanced NSCLC treatments with a particular emphasis on more recently approved agents.

\section{Search strategy}

In June 2014, we reviewed economic analyses of therapies for NSCLC identified by a MEDLINE, CRD, and HEED database search, using a disease-specific medical subject heading term ("lung neoplasms") and subheading ("economics"). Considering recent changes in practices, we limited this review to studies of patients managed since 2004 and to studies published between 2004 and 2014. We also made the same search, for the same period, in all medical economics publications identified in PubMed, restricted to papers with abstracts published in the English language. Analysis was restricted to chemotherapy drugs currently licensed in Europe and the United States for the first-line and maintenance treatment of patients with metastatic NSCLC. Economic analyses of small-cell lung cancer and other aspects of NSCLC, including smoking, screening, and diagnosis and staging procedures, are not reviewed here. Likewise, we did not select studies on the economic effect of supportive medications (growth factors, antiemetics, erythropoietins, etc). Relevant articles and extracts were selected and reviewed, and the reference lists from these sources were scanned for additional trials, as were the reference lists of relevant review articles. Outcomes were reported as stated in the studies without any yearly increment. The first step of this search strategy identified 2,274 published studies; 1,071 were published before 2004, 256 were in a non-English language, and 458 did not focus on approved chemotherapy drugs.

\section{Clinical guidelines for first-line and maintenance management for advanced NSCLC}

Clinical practice guidelines for first-line and maintenance management for advanced NSCLC have been drawn up by health care professionals and authorities in many countries and were recently updated. ${ }^{6}$
In patients with driver mutations (ie, epidermal growth factor receptor $[E G F R]$ gene mutations and anaplastic lymphoma kinase $[\mathrm{ALK}]$ translocations), first-line management recommended the use of an oral tyrosine kinase inhibitors (TKIs) or anti-ALK therapies such as erlotinib, gefitinib, or crizotinib until progression.

In patients with no or unknown driver mutations, good performance status (PS), and an age younger than 70 years, the recommendations were to use a platinum doublet therapy (cisplatin or carboplatin) with a third-generation drug (gemcitabine, docetaxel, paclitaxel, vinorelbine, and in cases with no squamous disease, pemetrexed) for four to six cycles. A meta analysis ${ }^{7}$ showed that there are no statistically significant differences in overall survival (OS) or progression-free survival (PFS) between any of the four third-generation chemotherapy doublets.

For patients with nonsquamous histology, a randomized Phase III trial suggested that cisplatin-pemetrexed (CisPem) increases OS compared with cisplatin-gemcitabine (CisGem). ${ }^{7}$ For patients with no squamous histology, bevacizumab may be associated with carboplatin paclitaxel (CarPac) in the United States and with any platinum doublet in Europe. ${ }^{7}$

For patients with a nonprogressive disease who remain in good health status after first-line chemotherapy, without residual toxicity, maintenance therapy can be proposed: continuous maintenance chemotherapy with bevacizumab or pemetrexed for no squamous cell carcinoma, with erlotinib, gefitinib, and pemetrexed (for no squamous cell carcinoma) as switch maintenance.

In elderly patients (older than 70 years) and patients with a PS of 2 who are unable to tolerate a platinum combination, a carboplatin combination or single-agent chemotherapy can be used.

\section{First-line chemotherapy in unselected advanced NSCLC patients}

Two recent reviews ${ }^{8,9}$ have reported on the main relevant results on this topic. We summarize here the main results of these reviews with a focus on the recently approved agents.

Making cost-effectiveness comparisons across available first-line chemotherapy treatments is limited by the comparability of the treatment populations and the short time frames. The majority of the reports have been conducted using a third-party payer perspective, taking into account only direct costs. ${ }^{8-12}$

When comparing CisGem and carboplatin paclitaxel, Neymark et $\mathrm{al}^{10}$ did not find any differences in survival 
between the two groups, but CisGem may reduce costs by approximately $€ 2,000$ per patient. The sensitivity analysis carried out on the base-case scenario did not lead to a change in the cost-effectiveness results. ${ }^{10} \mathrm{~A}$ retrospective costminimization analysis for Portugal ${ }^{12}$ of five doublet chemotherapy regimens from two Phase III trials in the treatment of advanced NSCLC showed that the least and the most costly chemotherapy regimens were, respectively, CisGem and cisplatin vinorelbine (CisVin). CisGem remained less costly in all sensitivity analyses. In Italy, ${ }^{13}$ the mean total treatment costs per patient were $€ 8,094$, $€ 11,203$, and $€ 9,320$ for the CisGem, CarPac, and CisVin regimens, respectively, and the authors concluded that $\mathrm{CisGem}$ was a cost-saving choice. Analyses taking into account the situation in several countries are rare. Schiller et $\mathrm{al}^{14}$ reported an updated cost-effectiveness of CisGem versus others cisplatin doublets, using the perspective of the national health services of five European countries (France, Germany, Italy, Spain, and the United Kingdom). Differences in total cost among the countries were primarily related to the different costs of chemotherapeutic agents and drug administration. CisGem was associated with lower total costs than CisPac and CarPac in all five countries. Compared with CisDoc, CisGem was associated with similar costs in Germany, France, and the United Kingdom and with lower costs in Italy and Spain. In the sensitivity analysis, inpatient versus outpatient administration had the greatest effect on overall cost differences.

For the CisPem doublet, the more complete study ${ }^{15}$ used a semi-Markov model to compare CisPem with CisGem, CarPac, and carboplatin-paclitaxel-bevacizumab (CarbPacBev). Data were extracted from a randomized controlled clinical trial comparing CisPem and CisGem, and as no head-to-head data were available, the authors used a mixed-treatment comparison model for the CisPem to CarPac or $\mathrm{CarbPacBev}$ comparisons. Medicare reimbursement rates were used to determine drug costs, and a retrospective claims database analysis was used to estimate other direct NSCLC-related costs. Regardless of histological subtype, using CisPem as first-line chemotherapy led to an incremental cost per life-year gained (LYG) of \$104,577 for CisPem compared with CisGem and \$231,291 for CisPem compared with CarPac. In the prespecified subset of patients with nonsquamous cell histology, the incremental cost per LYG was $\$ 83,537$ for CisPem compared with CisGem and $\$ 178,613$ for CisPem compared with CarPac. The incremental cost per LYG for CarbPacBev compared with CisPem was more than $\$ 300,000$. This analysis emphasizes the importance of histology in identifying the appropriate patient for CisPem first-line chemotherapy. Reasonable changes introduced by undertaking sensitivity analyses do not significantly change the base-case.

In a more recent, retrospective observational study ${ }^{16}$ evaluating the cost-effectiveness of first-line treatment of advanced nonsquamous NSCLC, costs for patients receiving CisPem were higher compared with costs for the CarPac doublet (difference, $\$ 21,841$ for PFS and $\$ 19,137$ for OS; $P \leq 0.05$ ), and patients receiving CisPem had lower mean costs compared with patients receiving $\mathrm{CarbPacBev}$ therapy (difference, $\$ 15,160$ for PFS and $\$ 19,946$ for OS; $P \leq 0.05)$.

In a model-based analysis, for all primary chemotherapy agents, ${ }^{9}$ use of carboplatin was associated with slightly higher costs than use of cisplatin. Outcomes vary between regimens, between docetaxel (best) and vinorelbine doublets (worst). In patients with squamous diseases, vinorelbine yielded the least patient benefit but is not the least expensive option. Paclitaxel doublets were consistently the minimum-cost options, and therefore represent the initial good value. The choice of preferred alternative main agents to paclitaxel generally favors docetaxel over gemcitabine, as docetaxel's greater effectiveness appears to outweigh the additional acquisition cost, although both drugs lie on the efficiency frontier. However, the difference in incremental quality-adjusted life-years (QALYs) gained between the treatments reflects only very marginal differences in benefit; in particular, the sensitivity of the results to the general level of drug prices relating to the choice of platinum compound indicates that in a competitive market, which has driven most generic prices down to very low levels, the price of drugs becomes less important than differences in the cost of drug administration and differences in the relative cost of adverse events.

Studies of doublets without platinum salts were rare. ${ }^{17,18}$ A study done in Greece comparing the docetaxel/gemcitabine combination with docetaxel monotherapy in untreated patients with advanced NSCLC ${ }^{18}$ showed an incremental cost per LYG of $€ 9,538$ when using the combination. The probability of being cost-effective was $91 \%$ at a threshold of $€ 20,000,97 \%$ at $€ 35,000$, and $98 \%$ at $€ 50,000$. The authors stated that the docetaxel/gemcitabine combination was a cost-effective treatment option relative to docetaxel monotherapy for patients with NSCLC in the Greek national health system setting. In contrast, in elderly patients with advanced disease, a recent review ${ }^{19}$ suggested that docetaxel monotherapy was cost-effective. In a recently published study based on a randomized trial done 10 years before, comparing CisGem, gemcitabine-vinorelbine, and CisVin, ${ }^{20}$ 
gemcitabine-vinorelbine was the most expensive regimen $(\$ 6,868)$, and CisVin was the cheapest $(\$ 4,650)$. Diagnostic and administration costs did not differ significantly among regimens; the principal cost drivers were toxicity and administration costs.

Oral treatments also have their place in this setting. A comparative cost-minimization of oral and intravenous chemotherapy for first-line treatment of NSCLC in the United Kingdom, from the National Health Service's point of view, showed that oral vinorelbine allows further hospital resource savings compared with other intravenous chemotherapies. ${ }^{21}$

\section{First-line target therapy in NSCLC patients with driver mutations NSCLC patients with EGFR mutation}

Advanced NSCLC patients with EGFR mutation had improved PFS with a TKI (erlotinib, gefitinib, and afatinib) treatment compared with platinum doublet therapy. ${ }^{22-24}$ A decision analytic mode ${ }^{25}$ showed that compared with the CarPac doublet, targeted therapy based on testing available tissue yielded an incremental cost-effectiveness ratio (ICER) of \$110,644 per QALY, and the rebiopsy strategy yielded an ICER of $\$ 122,219$ per QALY. Probabilistic sensitivity analysis revealed substantial uncertainty around these point estimates. With a willingness to pay of $\$ 100,000$ per QALY, the testing and the rebiopsy strategies were cost-effective, respectively, $58 \%$ and $54 \%$ of the time. Personalized therapy with an EGFR TKI was more favorable when the nontargeted chemotherapy regimen was more expensive. Compared with the triplet CarPemBev, ICERs were, respectively, \$25,547 and $\$ 44,036$ per QALY for the testing strategy and the rebiopsy strategy. These results appeared to be largely insensitive to varying the probability that a patient was EGFR-positive.

Another study developed a decision-analytic model to determine the ICER of EGFR testing and first-line treatment with gefitinib for patients who harbor activating EGFR mutations versus standard care, which includes first-line treatment with chemotherapy followed by gefitinib as second-line treatment. ${ }^{26}$ The model uses clinical and outcomes data from randomized clinical trials and societal costs from Singapore cancer centers. EGFR testing and first-line treatment with gefitinib is a dominant strategy compared with standard care. Because the primary savings result from not providing gefitinib to those who are not likely to benefit, this finding holds regardless of the prevalence of activating mutations. In a secondary analysis, first-line treatment with gefitinib was also dominant when compared with first-line chemotherapy in patients with activating EGFR mutations. A trial-based cost-effectiveness analysis of erlotinib alone versus platinumbased doublet chemotherapy as first-line therapy for Eastern Asian nonsquamous NSCLC. ${ }^{24}$ showed that Erlotinib monotherapy is more cost-effective from the Chinese health care system point of view. At a threshold of $\$ 96,884$, erlotinib had a $50 \%$ probability of being cost-effective.

An indirect treatment comparison and a cost-effectiveness analysis of erlotinib versus gefitinib as first-line treatment of EGFR activating mutation-positive NSCLC in Hong Kong, on the basis of four relevant Asian Phase III randomized controlled trials, ${ }^{27}$ resulted in a statistically significant PFS difference in favor of erlotinib (indirect treatment comparison hazard ratio, $0.33 ; 95 \%$ confidence interval, $0.19-0.58$; $P=0.0001)$. The ICER per LY and QALY was $\$ 39,431$ and $\$ 62,419$ for erlotinib versus gefitinib, respectively. For the authors, erlotinib appears cost-effective compared with gefitinib for first-line EGFR-mutated NSCLC patients.

\section{NSCLC patients with ALK translocation}

A recent paper analyzed the cost-effectiveness of echinoderm microtubule-associated protein-like 4 (EML4)-ALK fusion testing and first-line crizotinib treatment for patients with advanced ALK-positive nonsquamous NSCLC. ${ }^{28}$ Analysis was conducted using a Markov model from the Canadian Public Health (Ontario) perspective and a lifetime horizon. Molecular testing with first-line targeted crizotinib treatment in this population resulted in a gain of 0.011 QALY compared with standard care. The incremental cost was Canadian \$2,725 per patient, and the ICER was Canadian $\$ 255,970$ per QALY gained. Among patients with known EML4- $A L K$-positive advanced NSCLC, first-line crizotinib therapy provided 0.379 additional QALY, cost an additional $\$ 95,043$ compared with standard care, and produced an ICER of $\$ 250,632$ per QALY gained. The major driver of cost-effectiveness was drug price. The authors conclude that crizotinib treatment for $A L K$-positive NSCLC patients is not cost-effective in the setting of high drug costs and a low biomarker frequency. In this analysis, first-line standard care strategy was CisGem doublet without any maintenance, but the recommendations in this setting also allow pemetrexed as a cisplatin companion, as well as, after patients' selection, bevacizumab combined with a paclitaxel-carboplatin regimen. Continuation maintenance with bevacizumab or pemetrexed and switch maintenance with pemetrexed until progression are also acceptable options. Taking into account these costly drug options affects the costs and outcomes of standard care strategy. ${ }^{29}$ Unfortunately, in the sensitivity 
analysis, the cost of standard care chemotherapy was not tested.

\section{Bevacizumab as first-line and maintenance treatment}

Several analyses ${ }^{30-33}$ reported the cost-effectiveness of a bevacizumab-containing regimen with chemotherapy alone, for a maximum of six cycles and with the administration of bevacizumab as maintenance therapy in cases of response or disease stability, until disease progression or unacceptable toxicity.

Isla et $\mathrm{al}^{3}$ analyzed different schemes and observed a reduction of direct costs using bevacizumab. Bischoff et al, ${ }^{34}$ who focused their attention on the comparison of cisplatingemcitabine-bevacizumab with CisPem, obtained similar results. Stanisic et $\mathrm{al}^{35}$ analyzed indirect costs in addition to direct costs. They recorded a gain in terms of increased productivity (reduction of indirect costs) in the group of patients treated with regimens containing bevacizumab. This can be attributed to the increase of PFS and the improvement of quality of life. Klein et $\mathrm{al}^{36}$ analyzed first-line and maintenance regimens containing bevacizumab $15 \mathrm{mg} / \mathrm{kg}$ and concluded that these schemes are not cost-effective compared with pemetrexed-based regimens. Giuliani et $\mathrm{al}^{32}$ and Ahn et $\mathrm{al}^{30}$ concluded that schemes based on bevacizumab at a dose of $7.5 \mathrm{mg} / \mathrm{kg}$ in combination with CisGem are convenient compared with schemes based on CisPem. Finally, Goulart and Ramsey ${ }^{31}$ analyzed the cost-effectiveness of CisPem alone or in combination with bevacizumab at a dose of $15 \mathrm{mg} / \mathrm{kg}$. They concluded that regimens containing bevacizumab are not cost-effective. ${ }^{31}$ In fact, published studies are heterogeneous for different aspects, such as the dose of bevacizumab $(7.5$ or $15 \mathrm{mg} / \mathrm{kg})$ and the analysis of the direct costs (cost of drugs, costs related to the management of adverse events and increased survival) and indirect costs (costs related to loss of productivity in terms of work, cost of caregiver, etc). ${ }^{36}$

\section{Maintenance therapy (other than bevacizumab)}

Several economic studies were conducted on maintenance therapy. ${ }^{37,38}$ Maintenance gefitinib significantly prolonged PFS compared with placebo in patients from eastern Asia with locally advanced NSCLC with unknown EGFR mutations after four chemotherapeutic cycles of firstline platinum-based combination chemotherapy without disease progression. The cost-effectiveness of this maintenance strategy, ${ }^{38}$ from a Chinese health care system perspective, using a semi-Markov model, was an ICER of $\$ 19,214$ per QALY gained. The price of gefitinib is the most significant parameter that could reduce the incremental cost per QALY. ${ }^{39}$

For erlotinib compared with $\mathrm{BSC}$ as a maintenance therapy for advanced NSCLC, there were some disagreements. ${ }^{40-44}$ Two European studies concluded that erlotinib was cost-effective compared with BSC. ${ }^{44}$ Both studies extracted survival information directly from patient-level data in the SATURN (Sequential Tarceva in Unresectable NSCLC) trial, a Phase III clinical trial that compared erlotinib or placebo as first-line maintenance therapy: one was restricted to EGFRnegative patients, and the other was not. In contrast, in a US analysis (in which pemetrexed was the primary drug of interest), using indirect comparison instead of patient-level data, the authors estimated an ICER of $\$ 127,343$ per LYG in the comparison of erlotinib versus BSC, which appears as not cost-effective. ${ }^{36}$

For pemetrexed maintenance strategy, with the exception of the study by Greenhalgh et $a 1,{ }^{45}$ all studies indicated that pemetrexed is not cost-effective in this setting. From the perspective of the Swiss health care system, ${ }^{46}$ the ICER for pemetrexed maintenance was $€ 106,202$ per QALY. Uncertainties about the resource used and costs for BSC had a large influence on the cost-effectiveness calculation. From the perspective of the Chinese health care system, the ICERs of maintenance pemetrexed treatment after a CisPac strategy ${ }^{38}$ in a 1 or 2 year time horizon were $\$ 183,589$ and $\$ 126,353$ per QALY, respectively. The most sensitive influential variables were PFS health state utility, followed by proportion of patients with postdiscontinuation therapy in both groups. A paper presenting a summary of the evidence review group reported that ICER for pemetrexed maintenance treatment was $£ 47,000$ per QALY. ${ }^{5}$ From a US payer perspective, ${ }^{35}$ in the prespecified subset of patients with nonsquamous histology only, the ICER per LYG was $\$ 122,371$ for pemetrexed to observation and $\$ 150,260$ for pemetrexed to erlotinib. In all patients with advanced NSCLC, regardless of histologic subtype, using pemetrexed as maintenance therapy led to an ICER per LYG of \$205,597 compared with observation and $\$ 312,341$ compared with erlotinib. An adjusted, matched, indirect cross-market cost comparison of erlotinib versus pemetrexed for first-line maintenance treatment of patients with advanced NSCLC ${ }^{2}$ performed in France showed that acquisition costs was the main driver of total monthly per patient costs. Erlotinib appears to be a cost-saving treatment alternative to pemetrexed, producing comparable survival benefits at a lower cost. 


\section{Conclusion}

A number of economic analyses of first-line and maintenance therapies for NSCLC have been recently conducted. Differences in patient population, type of analysis, currency, year of valuation, time horizon, type of intervention, and included costs hinder direct comparisons of economic outcomes. The strength of these economic analyses is also limited, to varying degrees, by the retrospective nature of economic data collection in most studies, the lack of information on other health care costs (indirect, nonmedical, and intangible costs), and the limited time horizon, which often excludes terminal care, a costly phase of lung cancer management.

Today, significant advances continue to be made for NSCLC patients, and the treatment has become nuanced and specific for particular histological subtypes, clinical patient characteristics, and specific genetic mutations. ${ }^{47,48}$ This review showed that most recent clinical NSCLC guidelines were based on apparently no cost-effective strategies if we consider as acceptable threshold an ICER below \$50,000 per QALY gained. The limitations were that we need clinically relevant cost-effectiveness studies, probably at the country level, and a large debate involving public health organizations and pharmaceuticals companies, but also clinicians and patients, to challenge the rising of the cancer patient's management costs. Finally, careful assessment of the effect on quality of life of these new target therapies is mandatory.

\section{Disclosure}

In the last 5 years, $\mathrm{CC}$ has received fees for attending scientific meetings, speaking, organizing research, or consulting from AstraZeneca, Boehringer Ingelheim, GlaxoSmithKline, Hoffman la Roche, sanofi-aventis, Lilly, Novartis, and Amgen. PC received fees for attending scientific meetings from Hoffman la Roche and Lilly. IB received fees for attending scientific meetings or consulting from Hoffman la Roche. AV has received honoraria from Roche, Amgen, and Lilly and has received funding for clinical research from Astra-Zeneca, Chugaï, Lilly, Amgen, Roche, and Boehringer Ingelheim.

\section{References}

1. Gatta G, Rossi S, Capocaccia R. Cancer burden estimates and forecasts: uses and cautions. Tumori. 2013;99(3):439-443.

2. Chouaid C, Atsou K, Hejblum G, Vergnenegre A. Economics of treatments for non-small cell lung cancer. Pharmacoeconomics. 2009;27(2):113-125.

3. Isla D, González-Rojas N, Nieves D, Brosa M, Finnern HW. Treatment patterns, use of resources, and costs of advanced non-small-cell lung cancer patients in Spain: results from a Delphi panel. Clin Transl Oncol. 2011;13(7):460-471.
4. Neubauer MA, Hoverman JR, Kolodziej M, et al. Cost effectiveness of evidence-based treatment guidelines for the treatment of non-small-cell lung cancer in the community setting. J Oncol Pract. 2010;6(1):12-18.

5. Lewis JR, Lipworth WL, Kerridge IH, Day RO. The economic evaluation of personalised oncology medicines: ethical challenges. Med J Aust. 2013;199(7):471-473.

6. Besse B, Adjei A, Baas P, et al; Panel Members. 2nd ESMO Consensus Conference on Lung Cancer: non-small-cell lung cancer first-line/ second and further lines of treatment in advanced disease. Ann Oncol. 2014;25(8):1475-1484.

7. Brown T, Boland A, Bagust A, et al. Gefitinib for the first-line treatment of locally advanced or metastatic non-small cell lung cancer. Health Technol Assess. 2010;14(Suppl 2):71-79.

8. Bongers ML, Coupé VM, Jansma EP, Smit EF, Uyl-de Groot CA. Cost effectiveness of treatment with new agents in advanced nonsmall-cell lung cancer: a systematic review. Pharmacoeconomics. 2012;30(1):17-34.

9. Brown T, Pilkington G, Bagust A, et al. Clinical effectiveness and cost-effectiveness of first-line chemotherapy for adult patients with locally advanced or metastatic non-small cell lung cancer: a systematic review and economic evaluation. Health Technol Assess. 2013;17(31):1-278.

10. Neymark N, Lianes P, Smit EF, van Meerbeeck JP. Economic evaluation of three two-drug chemotherapy regimens in advanced non-small-cell lung cancer. Pharmacoeconomics. 2005;23(11):1155-1166.

11. Thongprasert S, Permsuwan U, Ruengorn C, Charoentum C, Chewaskulyong B. Cost-effectiveness analysis of cisplatin plus etoposide and carboplatin plus paclitaxel in a phase III randomized trial for nonsmall cell lung cancer. Asia Pac J Clin Oncol. 2011;7(4):369-375.

12. Pimentel FL, Bhalla S, Laranjeira L, Guerreiro M. Cost-minimization analysis for Portugal of five doublet chemotherapy regimens from two phase III trials in the treatment of advanced non-small cell lung cancer. Lung Cancer. 2006;52(3):365-371.

13. Novello S, Kielhorn A, Stynes G, et al; Italian Lung Cancer Project Investigators. Cost-minimisation analysis comparing gemcitabine/ cisplatin, paclitaxel/carboplatin and vinorelbine/cisplatin in the treatment of advanced non-small cell lung cancer in Italy. Lung Cancer. 2005;48(3):379-387.

14. Schiller J, Tilden D, Aristides M, et al. Retrospective cost analysis of gemcitabine in combination with cisplatin in non-small cell lung cancer compared to other combination therapies in Europe. Lung Cancer. 2004;43(1):101-112.

15. Klein R, Muehlenbein C, Liepa AM, Babineaux S, Wielage R, Schwartzberg L. Cost-effectiveness of pemetrexed plus cisplatin as first-line therapy for advanced nonsquamous non-small cell lung cancer. J Thorac Oncol. 2009;4(11):1404-1414.

16. Shah M, Winfree KB, Peterson P, Gruschkus SK, Eaddy M, Green MR. Cost effectiveness of first-line pemetrexed plus platinum compared with other regimens in the treatment of patients with nonsquamous non-small cell lung cancer in the US outpatient setting. Lung Cancer. 2013;82(1): 121-127.

17. Tada H, Matsui S, Kawahara M, Hosoe S, Hamada C, Fukushima M. Efficacy, toxicity and cost analysis for non-platinum triplet (gemcitabine and vinorelbine, followed by docetaxel) vs platinum-based chemotherapy in IIIB/IV non-small-cell lung cancer: single-institution experience. Eur J Cancer Care (Engl). 2008;17(2):120-126.

18. Maniadakis N, Fragoulakis V, Pallis A, Prezerakos P, Georgoulias V. Economic evaluation of docetaxel/gemcitabine versus docetaxel as frontline treatment of patients with advanced/metastatic non-small cell lung cancer in Greece. Lung Cancer. 2007;58(2):275-281.

19. Horn L, Visbal A, Leighl NB. Docetaxel in non-small cell lung cancer: impact on quality of life and pharmacoeconomics. Drugs Aging. 2007;24(5):411-428

20. Reaume MN, Leighl NB, Mittmann N, et al. Economic analysis of a randomized phase III trial of gemcitabine plus vinorelbine compared with cisplatin plus vinorelbine or cisplatin plus gemcitabine for advanced non-small-cell lung cancer (Italian GEMVIN3/NCIC CTG BR14 trial). Lung Cancer. 2013;82(1):115-120. 
21. Le Lay K, Myon E, Hill S, et al. Comparative cost-minimisation of oral and intravenous chemotherapy for first-line treatment of non-small cell lung cancer in the UK NHS system. Eur J Health Econ. 2007;8(2): 145-151.

22. Chouaid C, Le Caer H, Locher C, et al; GFPC 0504 Team. Cost effectivenes of erlotinib versus chemotherapy for first-line treatment of non small cell lung cancer (NSCLC) in fit elderly patients participating in a prospective phase 2 study (GFPC 0504). BMC Cancer. 2012;12(1):301.

23. Chouaid C, Le Caer H, Corre R, et al. Cost analysis of erlotinib versus chemotherapy for first-line treatment of non-small-cell lung cancer in frail elderly patients participating in a prospective phase 2 study (GFPC 0505). Clin Lung Cancer. 2013;14(2):103-107.

24. Wang S, Peng L, Li J, et al. A trial-based cost-effectiveness analysis of erlotinib alone versus platinum-based doublet chemotherapy as first-line therapy for Eastern Asian nonsquamous non-small-cell lung cancer. PLoS ONE. 2013;8(3):e55917.

25. Handorf EA, McElligott S, Vachani A, et al. Cost effectiveness of personalized therapy for first-line treatment of stage IV and recurren incurable adenocarcinoma of the lung. J Oncol Pract. 2012;8(5): 267-274.

26. de Lima Lopes G Jr, Segel JE, Tan DS, Do YK, Mok T, Finkelstein EA. Cost-effectiveness of epidermal growth factor receptor mutation testing and first-line treatment with gefitinib for patients with advanced adenocarcinoma of the lung. Cancer. 2012;118(4):1032-1039.

27. Lee VW, Schwander B, Lee VH. Effectiveness and cost-effectiveness of erlotinib versus gefitinib in first-line treatment of epidermal growth factor receptor-activating mutation-positive non-small-cell lung cancer patients in Hong Kong. Hong Kong Med J. 2014;20(3):178-186.

28. Djalalov S, Beca J, Hoch JS, et al. Cost effectiveness of EML4-ALK fusion testing and first-line crizotinib treatment for patients with advanced ALK-positive non-small-cell lung cancer. J Clin Oncol. 2014;32(10):1012-1019.

29. Chouaid C, Borget I, Vergnenegre A. Target therapies in non-small-cell lung cancer management: no cost-effective strategies? J Clin Oncol. Epub September 8, 2014.

30. Ahn MJ, Tsai CM, Hsia TC, et al. Cost-effectiveness of bevacizumabbased therapy versus cisplatin plus pemetrexed for the first-line treatment of advanced non-squamous NSCLC in Korea and Taiwan. Asia Pac J Clin Oncol. 2011;7(Suppl 2):22-33.

31. Goulart B, Ramsey S. A trial-based assessment of the cost-utility of bevacizumab and chemotherapy versus chemotherapy alone for advanced non-small cell lung cancer. Value Health. 2011;14(6):836-845.

32. Giuliani G, Grossi F, de Marinis F, Walzer S. Cost-effectiveness analysis of bevacizumab versus pemetrexed for advanced non-squamous NSCLC in Italy. Lung Cancer. 2010;69(Suppl 1):S11-S17.

33. Chien CR, Shih YC, Chun -Ru C, Ya-Chen TS. Economic evaluation of bevacizumab in the treatment of non-small cell lung cancer (NSCLC). Clinicoecon Outcomes Res. 2012;4:201-208.

34. Bischoff HG. Heigener DF, Walzer S, Nuijten M. Costs of bevacizumab and pemetrexed for advanced non-squamous NSCLC in Italy and Germany. Lung Cancer. 2010;1:S18-S23.
35. Stanisic S, Bischoff HG, Heigener DF, et al. Societal cost savings through bevacizumab-based treatment in non-small cell lung cancer (NSCLC). Lung Cancer. 2010;69(Suppl 1):S24-S30.

36. Klein R, Wielage R, Muehlenbein C, et al. Cost-effectiveness of pemetrexed as first-line maintenance therapy for advanced nonsquamous non-small cell lung cancer. J Thorac Oncol. 2010;5(8):1263-1272.

37. Zhu J, Li T, Wang X, et al. Gene-guided gefitinib switch maintenance therapy for patients with advanced EGFR mutation-positive non-small cell lung cancer: an economic analysis. BMC Cancer. 2013;13(1):39.

38. Zeng X, Peng L, Li J, et al. Cost-effectiveness of continuation maintenance pemetrexed after cisplatin and pemetrexed chemotherapy for advanced nonsquamous non-small-cell lung cancer: estimates from the perspective of the Chinese health care system. Clin Ther. 2013;35(1): 54-65

39. Zeng X, Li J, Peng L, et al. Economic outcomes of maintenance gefitinib for locally advanced/metastatic non-small-cell lung cancer with unknown EGFR mutations: a semi-Markov model analysis. PLoS ONE. 2014;9(2):e88881.

40. Dickson R, Bagust A, Boland A, et al. Erlotinib monotherapy for the maintenance treatment of non-small cell lung cancer after previous platinum-containing chemotherapy: a NICE single technology appraisal. Pharmacoeconomics. 2011;29(12):1051-1062.

41. Lyseng-Williamson KA. Erlotinib: a pharmacoeconomic review of its use in advanced non-small cell lung cancer. Pharmacoeconomics. 2010;28(1):75-92.

42. Nuijten MJ, de Castro Carpeño J, Chouaid C, et al. A cross-market cost comparison of erlotinib versus pemetrexed for first-line maintenance treatment of patients with locally advanced or metastatic non-small-cell lung cancer. Lung Cancer. 2012;76(3):465-471.

43. Walleser S, Ray J, Bischoff H, et al. Maintenance erlotinib in advanced nonsmall cell lung cancer: cost-effectiveness in EGFR wild-type across Europe. Clinicoecon Outcomes Res. 2012;4:269-275.

44. Vergnenègre A, Ray JA, Chouaid C, et al. Cross-market cost-effectiveness analysis of erlotinib as first-line maintenance treatment for patients with stable non-small cell lung cancer. Clinicoecon Outcomes Res. 2012;4: 31-37.

45. Greenhalgh J, McLeod C, Bagust A, et al. Pemetrexed for the maintenance treatment of locally advanced or metastatic non-small cell lung cancer. Health Technol Assess. 2010;14(Suppl 2):33-39

46. Matter-Walstra K, Joerger M, Kühnel U, Szucs T, Pestalozzi B, Schwenkglenks M. Cost-effectiveness of maintenance pemetrexed in patients with advanced nonsquamous-cell lung cancer from the perspective of the Swiss health care system. Value Health. 2012;15(1): 65-71.

47. Simonds NI, Khoury MJ, Schully SD, et al. Comparative effectiveness research in cancer genomics and precision medicine: current landscape and future prospects. J Natl Cancer Inst. 2013;105(13):929-936.

48. Uyl-de Groot CA, McDonnell J, Ten Velde G, Radice D, Groen HJ Cost-effectiveness of hypothetical new cancer drugs in patients with advanced small-cell lung cancer: results of a markov chain model. Ther Clin Risk Manag. 2006;2(3):317-323.
ClinicoEconomics and Outcomes Research

\section{Publish your work in this journal}

ClinicoEconomics \& Outcomes Research is an international, peerreviewed open-access journal focusing on Health Technology Assessment, Pharmacoeconomics and Outcomes Research in the areas of diagnosis, medical devices, and clinical, surgical and pharmacological intervention. The economic impact of health policy and health systems

\section{Dovepress}

organization also constitute important areas of coverage. The manuscript management system is completely online and includes a very quick and fair peer-review system, which is all easy to use. Visit http://www.dovepress.com/testimonials.php to read real quotes from published authors. 